\title{
Effect of Polyethylene Glycol on Crystallization Behavior of Acetaminophen
}

\author{
Zuzhao Zhou' ${ }^{\text {, Chengjun Jiang2* }}$ \\ ${ }^{1}$ Lianhe Chemical Technology (Taizhou) Co., Ltd., Taizhou, China \\ ${ }^{2}$ School of Biological and Chemical Engineering, Zhejiang University of Science and Technology, Hangzhou, China \\ Email: *jcj312@zust.edu.cn
}

How to cite this paper: Zhou, Z.Z. and Jiang, C.J. (2021) Effect of Polyethylene Glycol on Crystallization Behavior of Acetaminophen. Open Access Library Journal, 8: e7932. https://doi.org/10.4236/oalib.1107932

Received: September 7, 2021

Accepted: October 10, 2021

Published: October 13, 2021

Copyright $\odot 2021$ by author(s) and Open Access Library Inc.

This work is licensed under the Creative Commons Attribution International License (CC BY 4.0).

http://creativecommons.org/licenses/by/4.0/

\begin{abstract}
To investigate the effect of additives on the crystal morphology and crystal form. Acetaminophen crystals were prepared by cooling crystallization using ethanol as the solvent and adding different types of polymer compounds. The crystals of acetaminophen were characterized by X-ray powder diffraction, infrared spectroscopy, and optical microscopy. The results showed that polyethylene glycol was more effective in the inhibition of crystallization, which compared with tetrafluoroethylene, paraffin film and cellulose. In addition, although the increasement of polyethylene glycol content had no effect on the crystal form of acetaminophen, the crystal diameter diminished. This study provides reference for the stable production of crystal form I of acetaminophen.
\end{abstract}

\section{Subject Areas \\ Chemical Engineering, Technology}

\section{Keywords}

Acetaminophen, Polyethylene Glycol, Cooling Crystallization

\section{Introduction}

Pure crystals have been the focus of potential applications due to their unique properties, especially in the pharmaceutical industry where drugs are processed. The drug is subject to regulatory approval for single crystals or polymorphs. For the production of these drug substances, crystallization from solution is a critical unit operation, which bears great responsibility for obtaining high-purity chemicals and the final crystal form required for production [1] [2] [3]. The solubility, crystallinity and crystal properties of the active ingredients play a vital role in the 
development, production and formulation of drugs [4] [5]. Acetaminophen (also known as paracetamol) is a molecular organic compound and a well-known active ingredient in analgesic and antipyretic treatments. It is also used as an intermediate in the manufacture of culture medium, azo dyes and photographic chemicals [6] [7] [8] [9]. Acetaminophen has an amorphous state and three crystal forms, crystal form I, crystal form II, and crystal form III. When being used as a drug, crystal form III is basically not considered [10] [11]. Polymorphism refers to the ability of a molecule to exist in multiple different packaging arrangements in the solid state, and its different polymorphisms usually show significantly different physical and chemical properties [12]. The different crystal states of drug crystals make great differences in physicochemical indexes such as drug bioavailability, dissolution rate, and stability, which directly affect the drug bioavailability, safety, and efficacy. Therefore, the regulation of drug crystal form is the focus of research on drug quality and efficacy control. Additive molecules can not only affect the nucleation and growth process of drug molecules, but also affect the conversion process between crystal forms, thus affecting the crystal form and morphology of the final product [13] [14] [15] [16] [17]. In this paper, acetaminophen is taken as the research object, and the purpose is to study the influence of different additives on the crystallization behavior of acetaminophen. On this basis, the effect of the dosage of polyethylene glycol on acetaminophen will be further studied.

\section{Materials and Methods}

\subsection{Materials}

Acetaminophen were purchased from Energy Chemical (Shanghai, China).; Ethanol, polyethylene glycol (PEG); cellulose; parafilm; Poly tetrafluoroethylene (PTEF) were purchased from Shanghai Lingfeng Chemical Reagent Co., Ltd (Shanghai, China).

\subsection{Methods}

Using ethanol as the solvent, four acetaminophen solutions with the concentration of $0.250 \mathrm{~g} / \mathrm{mL}$ were prepared. Appropriate amounts of polyethylene glycol, poly tetrafluoroethylene, parafilm and cellulose were added into the four acetaminophen solutions respectively, labeled, crystallized for 8 hours, filtered, dried and characterized.

In studying the effect of polyethylene glycol on acetaminophen, $1 \mathrm{~mL}$ of 0.388 $\mathrm{g} / \mathrm{mL}$ polyethylene glycol was dropwise added into the different volumes of acetaminophen solution with the concentration of $0.250 \mathrm{~g} / \mathrm{mL}$.

The different volume $1.00 \mathrm{~mL}, 2.00 \mathrm{~mL}, 3.00 \mathrm{~mL}, 4.00 \mathrm{~mL}$ were marked as case A, case B, case C, case D.

\subsection{Characterization of Acetaminophen Cocrystal}

Fourier Transform Infrared Spectroscopy (FT-IR) spectra were recorded on a 
Perkin-Elmer Spectrum BX FTIR system, equipped with a deuterium triglycine sulfate detector. The scanrange was $500-4000 \mathrm{~cm}^{-1}$, using eight scans per spectrum with a resolution of $1 \mathrm{~cm}^{-1}$. Spectra were obtained in the transmission mode in $\mathrm{KBr}$ pellets.

Powder X-ray diffraction (PXRD) patterns were collected using Rigaku X-ray polycrystalline diffractometer. Samples were placed onto a silicon wafer slide. Generator settings were $40 \mathrm{kV}$ with a current of $40 \mathrm{~mA}$ used for the measurement. Data were collected $(\mathrm{n}=3)$ in the range $2 \theta=5^{\circ}$ to $40^{\circ}$ at a scanning rate of $5^{\circ} / \mathrm{min}$ with a scan rate of $0.04^{\circ} /$ time and a slit width of $6.0 \mathrm{~mm}$.

Microscopy was performed using Bai Datong wireless connection high-definition digital wifi microscope.

\section{Results and Discussion}

\subsection{Effect of Different Additives on Crystal Appearance of Acetaminophen}

Adding polyethylene glycol, tetrafluoroethylene, parafilm and cellulose to the paracetamol solution will crystallize paracetamol crystal particles of different sizes [13] [14] [15] [16] [17]. The results are shown in Figures 1-4.

As shown in Figures 1-4, Crystals of different sizes are crystallized on different polymer surfaces, the crystallization inhibition effect of polyethylene glycol on acetaminophen is the strongest, and the diameter of acetaminophen crystal precipitated after adding polyethylene glycol is the smallest. On the contrary, the crystallization inhibition effect of parafilm on acetaminophen is the weakest, and the diameter of acetaminophen crystal precipitated after adding parafilm is the largest, the size of the crystal can reach $6 \mathrm{~mm}$. Crystals on the surface of tetrafluoroethylene is slightly smaller than Crystals on the surface of parafilm. This is related to the properties of the polymer surface. Surfaces covered with polyethylene glycol (PEG; $\left.\mathrm{HO}-\left(\mathrm{CH}_{2}-\mathrm{CH}_{2}-\mathrm{O}\right)_{n}-\mathrm{H}\right)$ have been shown to great polarity

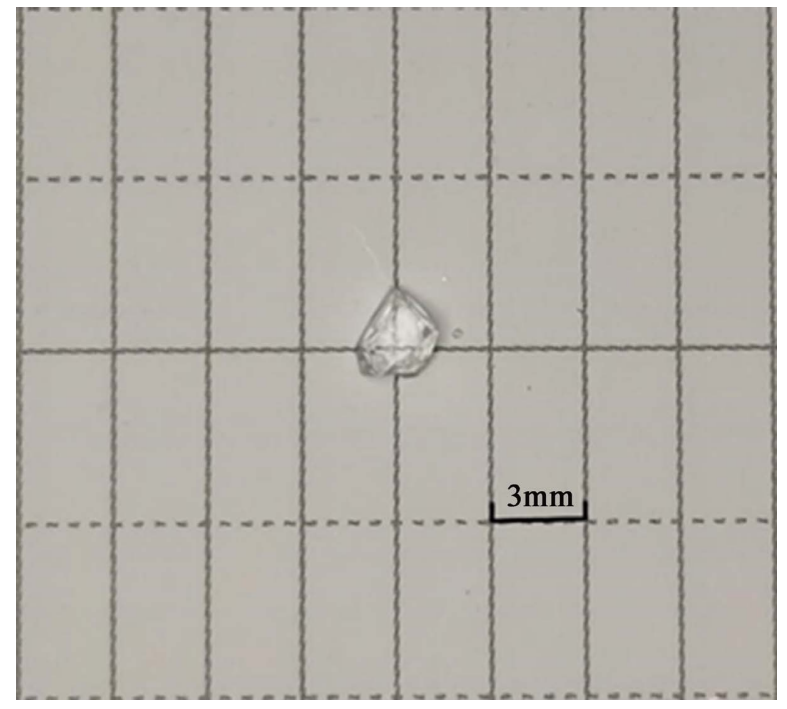

Figure 1. Crystals on the surface of PEG. 


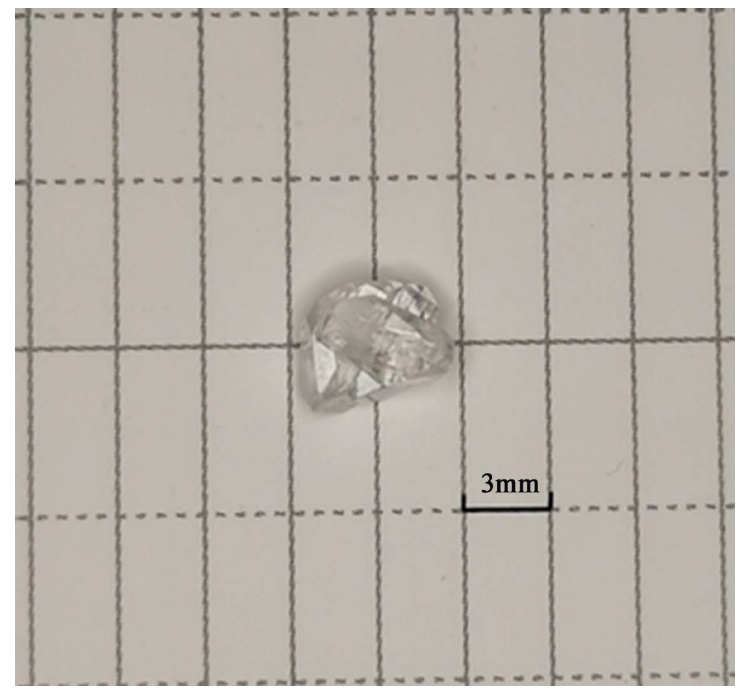

Figure 2. Crystals on the surface of PTEF.

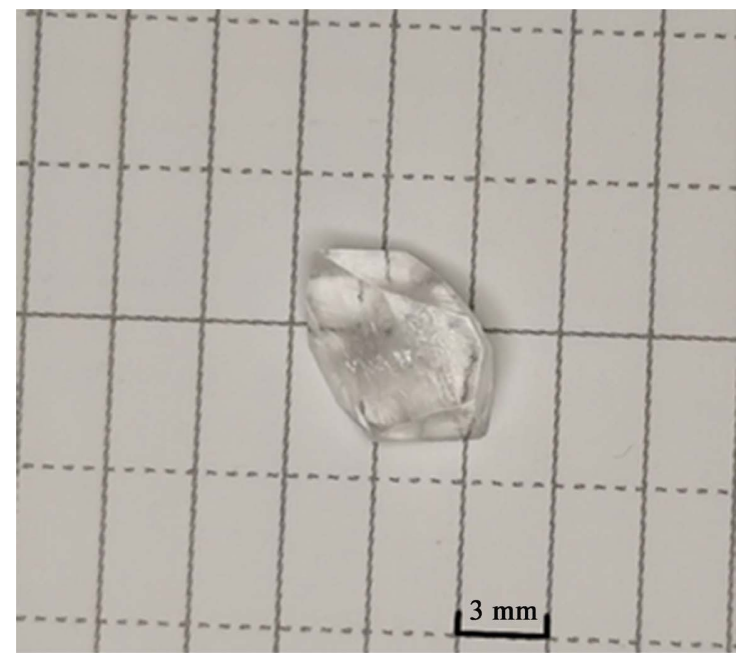

Figure 3. Crystals on the surface of parafilm.

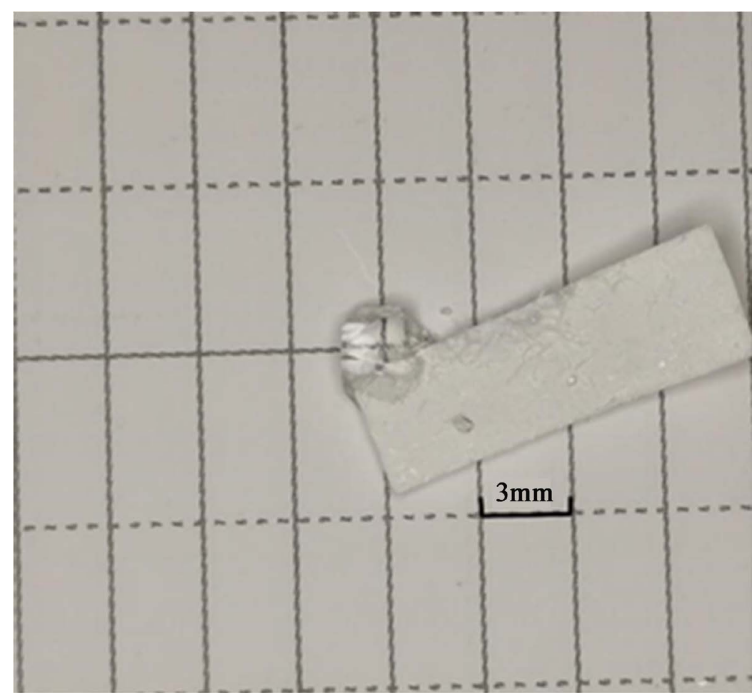

Figure 4. Crystals on the surface of cellulose. 
which can form hydrogen bonds with acetaminophen crystal surface [14], can change etching patterns in the direction of a-axi Parafilm is made of paraffin and primarily utilized to seal/protect containers in laboratories, polarity of the Parafilm surface is relatively the weakest.

\subsection{Effect of Dosage of Polyethylene Glycol on Crystallization Behavior of Acetaminophen}

\subsubsection{Powder X-Ray Diffraction Characterization of Acetaminophen}

The polymorphs were identified using powder X-ray diffraction (PXRD) or crystal morphology. The polymorphs and pseudopolymorphs of acetaminophen have different crystal morphologies, i.e., form I crystals have a platelike shape, form II crystals a needlelike shape, and trihydrate crystals a thin platelike shape [18] [19].

PXRD patterns of acetaminophen crystals as shown in Figure 5. The $2 \theta$ angles of crystal PXRD have characteristic absorption peaks at about $12.1^{\circ}, 13.8^{\circ}, 15.5^{\circ}$, $16.7^{\circ}, 18.2^{\circ}, 18.9^{\circ}, 20.4^{\circ}, 20.8^{\circ}, 23.4^{\circ}, 24.3^{\circ}, 26.5^{\circ}, 27.1^{\circ}, 29.0^{\circ}, 32.6^{\circ}, 36.9^{\circ}, 38.5^{\circ}$ and $40.5^{\circ}$, which matched with the PXRD pattern of type I crystal [19].

\subsubsection{Infrared Characterization of Acetaminophen}

Using ethanol as the solvent, and adding different amounts of polyethylene glycol, the infrared spectrum of the crystal was obtained as shown in Figure 6.

As shown in Figure 6, the sample in the $3325 \mathrm{~cm}^{-1}, 3108 \mathrm{~cm}^{-1}, 3163 \mathrm{~cm}^{-1}$ or so had free hydroxyl stretching vibration absorption peak, the secondary amide nitrogen hydrogen stretching vibration absorption peak and the corresponding associating fit existence. At about $1652 \mathrm{~cm}^{-1}, 1610 \mathrm{~cm}^{-1}, 1563 \mathrm{~cm}^{-1}, 1506 \mathrm{~cm}^{-1}$, there were stretching vibration absorption peaks of aromatic ring skeleton structure,

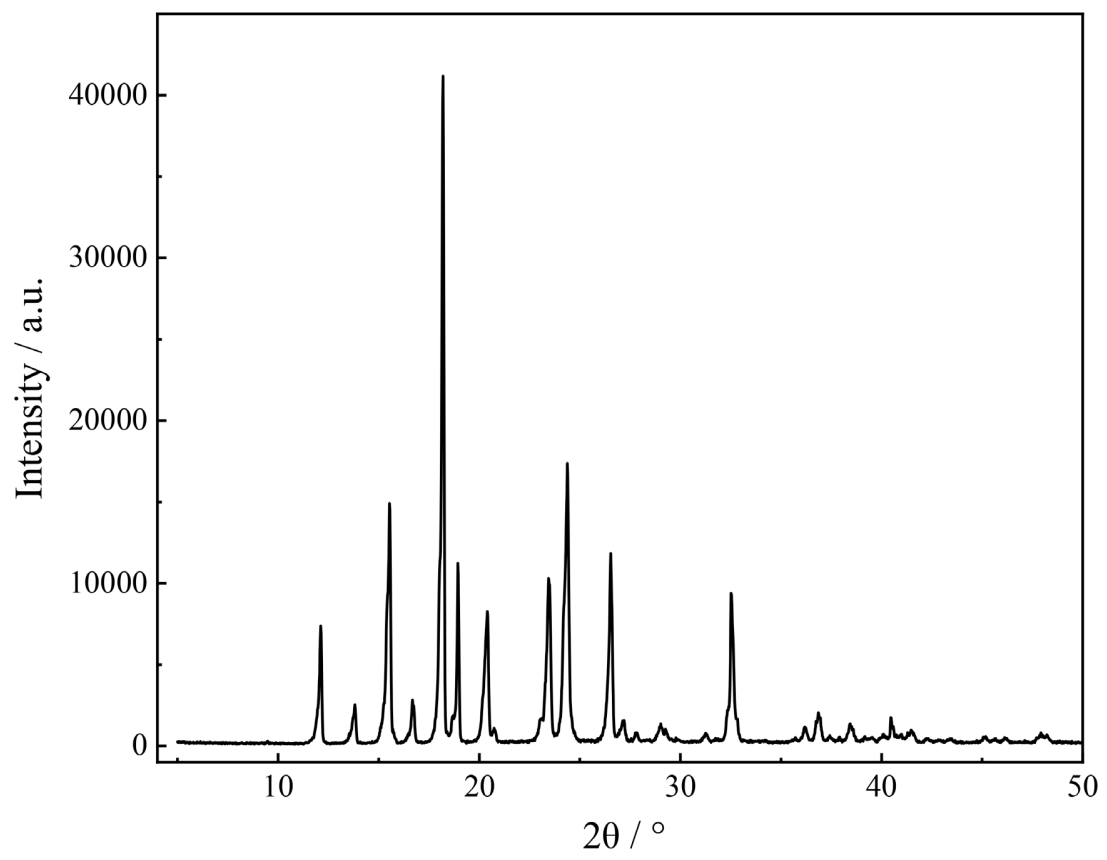

Figure 5. PXRD patterns of acetaminophen crystals. 


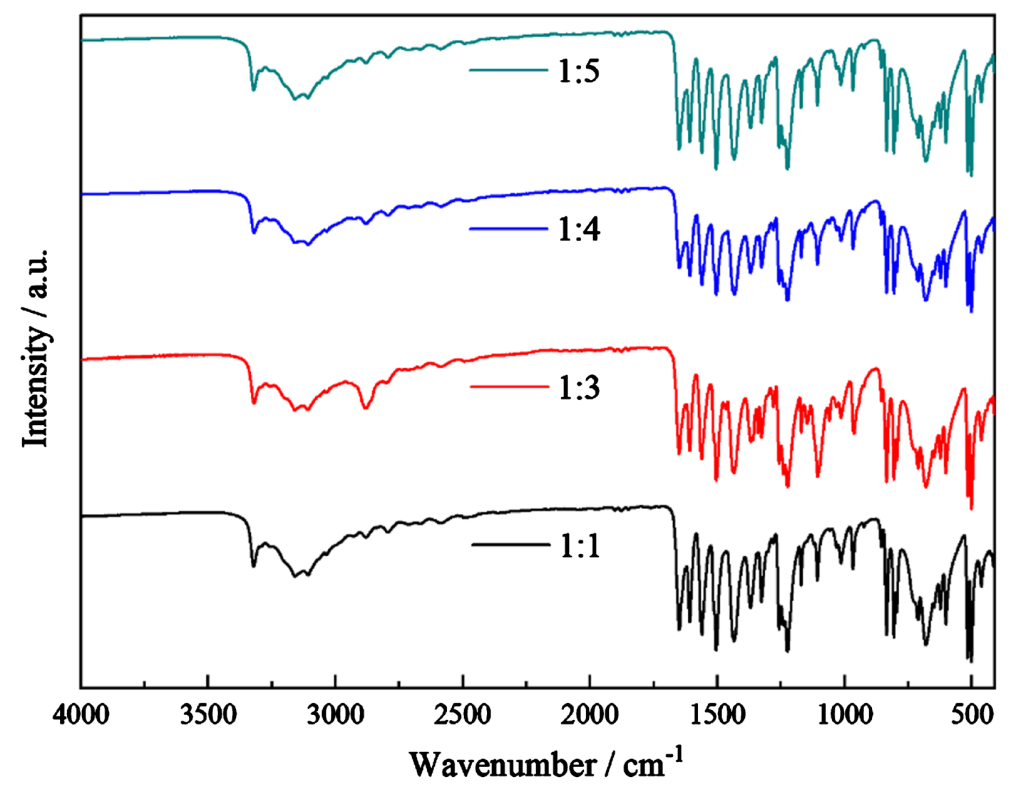

Figure 6. Comparison of infrared spectra of acetaminophen crystal.

stretching vibration of carbon-oxygen double bond in secondary amide, and bending vibration absorption peaks of nitrogen and hydrogen. About $1438 \mathrm{~cm}^{-1}$ and $1373 \mathrm{~cm}^{-1}$ were vibration absorption peaks of methyl deformation. There were stretching vibration absorption peaks of aryl carbon and nitrogen at around $1328 \mathrm{~cm}^{-1}, 1325 \mathrm{~cm}^{-1}$, and $1259 \mathrm{~cm}^{-1}$. At about $1227 \mathrm{~cm}^{-1}, 1171 \mathrm{~cm}^{-1}, 1108 \mathrm{~cm}^{-1}$ and $1016 \mathrm{~cm}^{-1}$, there were the stretching vibration absorption peaks of hydroxyl carbon oxygen and the stretching vibration absorption peaks of alkyl carbon and nitrogen. There were absorption peaks of deformation vibration and bending vibration of the carbon-hydrogen bond on aromatic hydrocarbons at about 967 $\mathrm{cm}^{-1}, 835 \mathrm{~cm}^{-1}, 807 \mathrm{~cm}^{-1}, 714 \mathrm{~cm}^{-1}, 683 \mathrm{~cm}^{-1}, 625 \mathrm{~cm}^{-1}, 604 \mathrm{~cm}^{-1}, 517 \mathrm{~cm}^{-1}, 501$ $\mathrm{cm}^{-1}$.

There was no significant difference in the infrared spectrum. The change of PEG concentration did not affect the crystal form.

\subsubsection{Optical Microscopic Characterization of Acetaminophen Crystal Form}

As shown in Figure 7, comparison of the crystals in the optical microscope shows that the crystal morphology of acetaminophen formed varies with the amount of polyethylene glycol. Supersaturation is the main driving force in the process of crystal growth [20]. The larger the supersaturation of the solution, the greater the driving force generated in the crystallization process, and the greater the nucleation rate and growth rate of the crystal [20]. The addition of polyethylene glycol to acetaminophen increases the solubility of acetaminophen in ethanol. However, the solubility of acetaminophen in ethanol is not completely proportional to the dosage of polyethylene glycol. When the polyethylene glycol reaches a specific concentration, acetaminophen is difficult to form a supersaturated state in ethanol, so no crystal is precipitated. 

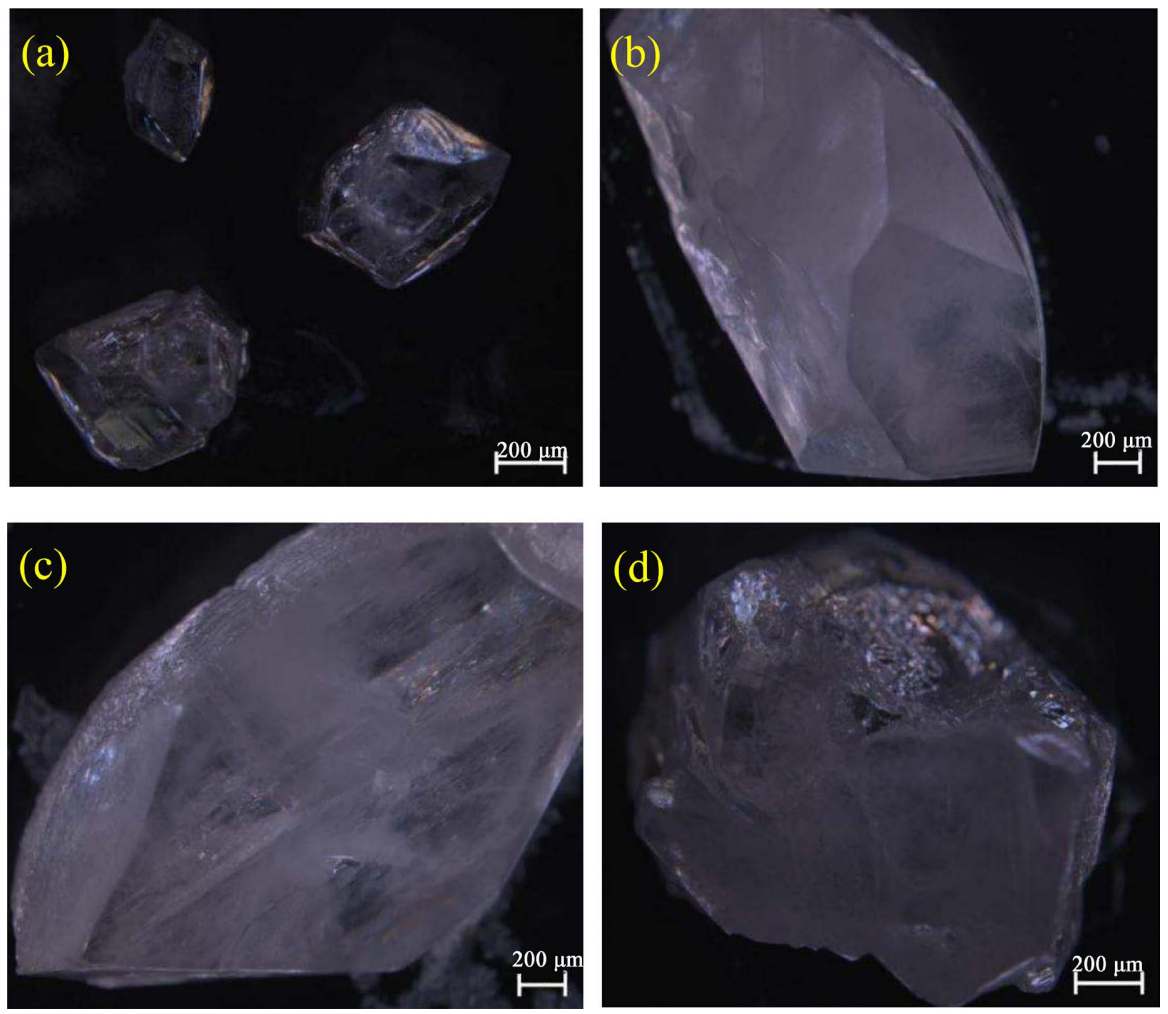

Figure 7. Photographs of acetaminophen crystals.

\section{Conclusion}

Acetaminophen crystals precipitated by adding different types of high molecular polymers in ethanol have different morphologies. Among them, acetaminophen crystals precipitated by adding polyethylene glycol have the smallest amount and the smallest crystal diameter, so it has the strongest inhibition on crystallization of acetaminophen. Therefore, the effect of the amount of polyethylene glycol on the crystallization behavior of acetaminophen was further studied. Polyethylene glycol is a nonionic surfactant with water solubility and polar oligomer. It can be directionally adsorbed on the surface of acetaminophen to make it have charge characteristics and form an adsorption layer to improve the dispersibility of the product [21]. Therefore, polyethylene glycol has a good anti-agglomeration effect on acetaminophen. This study is expected to provide a reference for the stable production of acetaminophen I. However, the mechanism that acetaminophen is difficult to reach the supersaturation state in ethanol solvent under the specific concentration of polyethylene glycol requires further research.

\section{Conflicts of Interest}

The authors declare no conflicts of interest.

\section{References}

[1] Ó'Ciardhá, C.T., Frawley, P.J. and Mitchell, N.A. (2011) Estimation of the Nucleation Kinetics for the Anti-Solvent Crystallisation of Paracetamol in Methanol/Water 
Solutions. Journal of Crystal Growth, 328, 50-57. https://doi.org/10.1016/j.jcrysgro.2011.06.017

[2] Tung, H.H., Paul, E.L., Midler, M. and McCauley, J.A. (2009) Crystallization of Organic Compounds: An industrial Perspective. John Wiley \& Sons Publishers, Hoboken. https://doi.org/10.1002/9780470447796

[3] Kuvadia, Z.B. and Doherty, M.F. (2011) Spiral Growth Model for Faceted Crystals of Non-Centrosymmetric Organic Molecules Grown from Solution. Crystal Growth \& Design, 11, 2780-2802.

[4] Tedesco, E., Giron, D. and Pfeffer, S. (2001) Crystal Structure Elucidation and Morphology Study of Pharmaceuticals in Development. CrystEngComm, 67, 393-400. https://doi.org/10.1039/B202427F

[5] Keraliya, R.A., Soni, T.G., Thakkar, V.T. and Gandhi, T.R. (2010) Effect of Solvent on Crystal Habit and Dissolution Behavior of Tolbutamide by Initial Solvent Screening. Dissolution Technologies, 17, 16-21. https://doi.org/10.14227/DT170110P16

[6] Al-Mayahi, A.M.W. (2016) Effect of Silicon (Si) Application on Phoenix Dactylifera L. Growth under Drought Stress Induced by Polyethylene Glycol (PEG) in Vitro. American Journal of Plant Sciences, 7, 1711-1728. https://doi.org/10.4236/ajps.2016.713161

[7] Boerrigter, S.X.M., Cuppen, H.M., Ristic, R.I., Sherwood, J.N., Bennema, P. and Meekes, H. (2002) Explanation for the Supersaturation-Dependent Morphology of Monoclinic Paracetamol. Crystal Growth \& Design, 2, 357-361. https://doi.org/10.1021/cg020012r

[8] Omar, W., Al-Sayed, S., Sultan, A. and Ulrich, J. (2008) Growth Rate of Single Acetaminophen Crystals in Supersaturated Aqueous Solution under Different Operating Conditions. Crystal Research and Technology, 43, 22-27. https://doi.org/10.1002/crat.200710995

[9] Granberg, R.A. and Rasmuson, Å.C. (1999) Solubility of Paracetamol in Pure Solvents. Journal of Chemical \& Engineering Data, 44, 1391-1395. https://doi.org/10.1021/je990124v

[10] Ambike, A.A., Mahadik, K.R. and Paradkar, A. (2004) Stability Study of Amorphous Valdecoxib. International Journal of Pharmaceutics, 282, 151-162. https://doi.org/10.1016/j.ijpharm.2004.06.009

[11] Espeau, P., Céolin, R., Tamarit, J., Perrin, M.A., Gauchi, J.P. and Leveiller, F. (2010) Polymorphism of Paracetamol: Relative Stabilities of the Monoclinic and Orthorhombic Phases Inferred from Topological Pressure-Temperature and TemperatureVolume Phase Diagrams. Journal of Pharmaceutical Sciences, 94, 524-539. https://doi.org/10.1002/jps.20261

[12] Agnew, L.R., McGlone, T., Wheatcroft, H.P., Robertson, A., Parsons, A.R. and Wilson, C.C. (2017) Continuous Crystallization of Paracetamol (Acetaminophen) Form II: Selective Access to a Metastable Solid Form. Crystal Growth \& Design, 17, 2418 2427. https://doi.org/10.1021/acs.cgd.6b01831

[13] Golitsyn, Y., Pulst, M., Samiullah, M.H., Busse, K., Kressler, J. and Reichert, D. (2019) Crystallization in PEG Networks: The Importance of Network Topology and Chain Tilt in Crystals. Polymer, 165, 72-82. https://doi.org/10.1016/j.polymer.2019.01.018

[14] Sun, Y., Zhu, L., Wu, T., Cai, T., Gunn, E.M. and Yu, L. (2012) Stability of Amorphous Pharmaceutical Solids: Crystal Growth Mechanisms and Effect of Polymer Additives. The AAPS Journal, 14, 380-388. https://doi.org/10.1208/s12248-012-9345-6 
[15] Thompson, C., Davies, M.C., Roberts, C.J., Tendler, S.J.B. and Wilkinson, M.J. (2004) The Effects of Additives on the Growth and Morphology of Paracetamol (Acetaminophen) Crystals. International Journal of Pharmaceutics, 280, 137-150. https://doi.org/10.1016/j.ijpharm.2004.05.010

[16] Wen, H., Morris, K.R. and Park, K. (2005) Hydrogen Bonding Interactions between Adsorbed Polymer Molecules and Crystal Surface of Acetaminophen. Journal of Colloid and Interface Science, 290, 325-335.

https://doi.org/10.1016/j.jcis.2005.04.049

[17] Wen, H., Morris, K.R. and Park, K. (2008) Synergic Effects of Polymeric Additives on Dissolution and Crystallization of Acetaminophen. Pharmaceutical Research, 25, 349-358. https://doi.org/10.1007/s11095-007-9468-0

[18] del Río, J.R.M. and Rousseau, R.W. (2006) Batch and Tubular-Batch Crystallization of Paracetamol: Crystal Size Distribution and Polymorph Formation. Crystal Growth \& Design, 6, 1407-1414. https://doi.org/10.1021/cg060025v

[19] Nichols, G. and Frampton, C.S. (1998) Physicochemical Characterization of the Orthorhombic Polymorph of Paracetamol Crystallized from Solution. Journal of Pharmaceutical Sciences, 87, 684-693. https://doi.org/10.1021/js970483d

[20] Trasi, N.S., Oucherif, K.A., Litster, J.D. and Taylor, L.S. (2015) Evaluating the Influence of Polymers on Nucleation and Growth in Supersaturated Solutions of Acetaminophen. CrystEngComm, 17, 1242-1248. https://doi.org/10.1039/C4CE02179G

[21] Strauss, V., Margraf, J.T., Dolle, C., Butz, B., Nacken, T.J., Walter, J., et al. (2014) Carbon Nanodots: Toward a Comprehensive Understanding of Their Photoluminescence. Journal of the American Chemical Society, 136, 17308-17316. https://doi.org/10.1021/ja510183c 